\title{
The nucleotidohydrolases DCTPP1 and dUTPase are involved in the cellular response to the DNA-demethylating drug decitabine
}

Cristina E. Requena ${ }^{1}$, Guiomar Pérez-Moreno ${ }^{1}$, András Horváth ${ }^{2}$, Beáta G. Vértessy ${ }^{2,3}$, Luis M. Ruiz-Pérez ${ }^{1}$, Dolores González-Pacanowska ${ }^{1, *}$ and Antonio E. Vidal ${ }^{1, *}$

${ }^{1}$ Departmento de Bioquímica y Farmacología Molecular, Instituto de Parasitología y Biomedicina Lopez-Neyra, Consejo Superior de Investigaciones Científicas (CSIC), Armilla (Granada), 18016, Spain

${ }^{2}$ Institute of Enzymology, Research Center for Natural Sciences, Hungarian Academy of Science, H-1117 Budapest, Hungary

${ }^{3}$ Department of Applied Biotechnology and Food Sciences, University of Technology and Economics, H-1111 Budapest, Hungary

Running title: Role of dUTPase and dCTPase on decitabine action Keywords: DCTPP1, XTP3-TPA, dUTPase, nucleotide pool, decitabine

\section{GRANT SUPPORT}

This work was supported by Ministerio de Economía y Competitividad [Plan Nacional de Investigación, SAF2011-27860 to A.E. Vidal, SAF2013-48999-R to D. GonzálezPacanowska]; Junta de Andalucía [BIO-199, P12-BIO-2059] (to D. GonzálezPacanowska); Hungarian National Research, Development and Innovation Office [NK84008 and K-109486]; and ICGEB [CRP HUN14-01] (to B.G. Vértessy).

The authors disclose no potential conflicts of interest. 
*Corresponding author: Antonio E. Vidal, Instituto de Parasitología y Biomedicina Lopez-Neyra (CSIC) Avda. del Conocimiento s/n, Armilla (Granada) 18016 Spain. Tel: +34 (958) 181 656; Fax: +34 (958) 181 632; Email: avidal@ipb.csic.es Correspondence may also be addressed to: Dolores González-Pacanowska, Instituto de Parasitología y Biomedicina Lopez-Neyra (CSIC) Avda. del Conocimiento s/n, Armilla (Granada) 18016 Spain. Tel: +34 (958) 181 631; Fax: +34 (958) 181 632; Email: dgonzalez@,ipb.csic.es

Number of Figures: 7 


\begin{abstract}
Decitabine (5-aza-2'-deoxycytidine, aza-dCyd) is a potent DNA-demethylating and genotoxic drug used clinically for the treatment of myelodysplastic syndromes and acute myeloid leukemia. On the other hand, DCTPP1 and dUTPase are two "house cleaning" nucleotidohydrolases involved in the elimination of non-canonical nucleotides. Here we show that exposure of HeLa cells to aza-dCyd up-regulates the expression of several pyrimidine metabolic enzymes including DCTPP1, dUTPase, dCMP deaminase and thymidylate synthase thus suggesting their contribution to the cellular response to this anticancer nucleoside. We present several lines of evidence supporting that, in addition to the formation of aza-dCMP, an alternative cytotoxic mechanism for decitabine involving the formation of aza-dUMP, a potential thymidylate synthase inhibitor. Indeed, dUTPase or DCTPP1 down-regulation enhanced the cytotoxic effect of aza-dCyd producing an accumulation of nucleosides triphosphate containing uracil as well as uracil misincorporation and double-strand breaks in genomic DNA. Moreover, DCTPP1 hydrolyzes the triphosphate form of decitabine with similar kinetic efficiency than its natural substrate dCTP and prevents decitabineinduced global DNA demethylation. We propose that DCTPP1 and dUTPase nucleotidohydrolases are key factors in decitabine mode of action with potential value as predictive markers of the clinical response or as enzymatic targets to improve decitabine-based chemotherapy.
\end{abstract}




\section{INTRODUCTION}

Nucleoside analogues are effective antimetabolites commonly used as anticancer agents. The cytidine analog decitabine (5-aza-2'-deoxycytidine, aza-dCyd) is a DNA demethylating agent and a potent anticancer drug used clinically for the treatment of myelodysplastic syndromes (MDS) and acute myeloid leukemia (1,2). Aza-dCyd is metabolically activated in vivo through consecutive phosphorylations, into aza-dCTP that is readily incorporated into DNA and extended by the DNA polymerase (3). Once in DNA, aza-dCyd acts as a suicidal substrate by covalently trapping DNA methyltransferase (DNMT) molecules that attempt to initiate cytosine methylation. The resulting DNA-protein cross-links trigger the proteosomal degradation machinery and lead to the depletion of the DNA methylation activities of the cell. Consequently, the replacement of deoxycytidine ( $\mathrm{dCyd}$ ) by aza-dCyd results in hypomethylation at the promoter DNA regions and the reactivation of epigenetically repressed genes (4). The transcriptional activation of tumor suppressor genes which are aberrantly silenced in cancer cells constitutes the basic principle of the epigenetic cancer therapy.

The extent to which the in vivo antitumor properties of decitabine and the clinical response to decitabine treatment depend on epigenetic activities remains unclear. It has not been possible to establish a definitive correlation between the inhibition of cell proliferation and clinical response with the reversal of methylation and gene reexpression (5). In fact, it is generally agreed that decitabine has dual effects on neoplastic cells in a dose-dependent manner; at low doses, cells survive but reactivation of genes that control proliferation may lead to differentiation, cell cycle arrest and increased apoptosis (6). At high doses, decitabine induces genome-wide DNA damage and cytotoxicity (7). Most of the DNA damage arises from the DNA-DNMT crosslinks which can block DNA synthesis and induce directly or indirectly DNA double-strand 
breaks, eventually leading to cell death (8). Decitabine has also been reported to enhance mutagenesis, mainly point mutations and genome rearrangements most likely due to protein-DNA crosslinks $(9,10)$. The biochemical pathway that initiates repair of DNA-DNMT adducts has not been yet described in detail, however, it may involve DNA double-strand break repair factors at its late stages. It is well established that decitabine treatment induces the activation of a specific DNA damage response that includes the phosphorylation of histone H2AX (7). Additionally, the cytotoxic and mutagenic properties of decitabine may be partially derived from its intrinsic chemical instability once it is incorporated into DNA or by the accumulation of DNA repair intermediates (3). Recently, it has been proposed that the base excision repair mechanism (BER) might be initiating the repair of decitabine-induced DNA base lesions although the precise nature of the damage or the DNA glycosylases involved remains unknown (11).

The all-alpha NTP pyrophosphatase DCTPP1 (dCTP pyrophosphatase 1) is a recently characterized pyrophosphohydrolase that contributes to the homeostasis of the dNTP pool in human cells by controlling the levels of $\mathrm{dCTP}$, one of its major substrates. DCTPP1 can also hydrolyze C5-modified dNTPs such as 5-halogenated, 5-methyl and 5-formyl deoxycytidines, and therefore may have an additional 'house-cleaning' function (12). The enzyme deoxyuridine triphosphate nucleotidohydrolase (dUTPase) also plays two roles, providing dUMP for de novo biosynthesis of dTTP and sanitizing the deoxynucleotide pool by the specific removal of dUTP (13-15).

In this study, we have investigated the role of the nucleotidohydrolases DCTPP1 and dUTPase in the metabolic response to decitabine. We found that DCTPP1 downregulation increased aza-dCyd-induced toxicity in HeLa and MRC-5 cells at a wide range of doses. DCTPP1 can hydrolyze the activated form of decitabine, 5-aza-2'- 
deoxycytidine-5'-triphoshate and might constitute a novel route in the detoxification of this DNA methylation inhibitor. In support of this notion, the analysis of the molecular mechanism behind the growth inhibiting effect revealed that the absence of normal levels of DCTPP1 had an impact on global DNA demethylation levels. On the other hand, dUTPase depletion also sensitized cells to aza-dCyd and promoted a significant accumulation of dUTP in the nucleotide pool and uracil and double-strand breaks in the genomic DNA as a consequence of the treatment. A similar DNA damage signature was found in DCTPP1-deficient cells exposed to decitabine suggesting the additional implication of this enzyme in an alternate mode of action for decitabine in which the metabolism of aza-dCyd may ultimately lead to the genotoxic accumulation of dUTP in the cellular pool. 


\section{MATERIAL AND METHODS}

\section{Nucleotides, nucleosides and antibodies.}

Decitabine (aza-dCyd), cytarabine (ara-Cyd) and 2'-deoxycytidine (dCyd) were purchased from Sigma; 5-aza-2'-deoxycytidine-5' -triphosphate (aza-dCTP), ara-CTP, 2 '-difluorocytidine-5'-triphosphate (dF-CTP) and 2',3'-dideoxycytidine-5'-triphosphate (ddCTP) were obtained from Jena Bioscience. Anti-DCTPP1 (12) and anti-DUT (this work) are anti-rabbit polyclonal antibodies generated in our laboratory. dCMP deaminase (DCTD), thymidylate kinase (TMPK), thymidylate synthase (TS) and thymidine kinase (TK) detection was carried out with anti-rabbit polyclonal antibodies from Santa Cruz Biotechnologies Inc. Cytidine deaminase (CDA) and deoxycytidine kinase $(\mathrm{dCK})$ were detected with anti-rabbit anti-CDA and anti-mouse anti-dCK antibodies respectively (Abcam). Anti- $\alpha$-tubulin was purchased from Ab Frontier.

\section{Cell cultures and transfections}

Two wild-type p53-expressing cell lines were used, the normal human fibroblast line MRC-5 (ATCC, CCL 171) and the tumor epithelial line HeLa (ATCC, CCL-2). The cell lines were not passaged for more than 6 months following purchase. MRC-5 was cultured in Eagle's Minimum Essential Medium (Gibco, Life Technologies) with $2 \mathrm{mM}$ L-glutamine (GlutaMAX ${ }^{\mathrm{TM}}$, Gibco, Life technologies) and HeLa cell line was cultured in Dulbecco's Modified Eagle's Medium (PAA, GE Healthcare) supplemented with 1X MEM Non-essential Amino Acids Solution (Gibco, Life technologies) and 2 mM Lglutamine. Media were supplemented with $10 \%$ fetal bovine serum (FBS), $100 \mathrm{U} \mathrm{ml}^{-1}$ penicillin and $100 \mu \mathrm{g} \mathrm{m}^{-1}$ streptomycin (Gibco, Life technologies). Cell lines were cultured in a humidified atmosphere of $5 \% \mathrm{CO}_{2}$ at $37^{\circ} \mathrm{C}$. 
DCTPPI and $d U T P a$ se silencing were carried out with small interfering RNA (siRNA) oligonucleotide pool (ON-TARGETplus smart pool, Dharmacon). The DCTPP1specific siRNAs have been previously validated (12). For dUTPase silencing, the following mRNA sequences were targeted: 5'-GCUCAUUUGCGAACGGAUU-3', 5'UGUAGGAGCUGGUGUCAUA-3'，5'-UAGAGGAAAUGUUGGUGUU-3' and 5'UGCCUAUGAUUACACAAUA-3. The negative control consisted of four nontargeting siRNAs oligonucleotides (ON-TARGETplus non-targeting pool, Dharmacon). Transient transfections were carried out according to the manufacturer's instructions.

\section{Proliferation and clonogenic assays.}

$10^{3}$ cells were exposed to increasing doses $(0-1 \mathrm{mM})$ of decitabine, deoxycytidine or cytarabine at $37^{\circ} \mathrm{C}$ for 24 or $72 \mathrm{~h}$ for Hela and MRC-5 respectively. Incubation times were decided based on differences in cell proliferation and sensitivity to decitabine. The medium was exchanged and the drug replenished every $24 \mathrm{~h}$. Viable cell number was determined by Resazurin (Sigma) reduction. For the clonogenic assay, cells were seeded in a 6-well plate at a density of 300 cells per well and treated with decitabine. After completion of the treatments, fresh growth medium was added to the wells and cells were left to proliferate for 7 (Hela) or 12 (MRC-5) additional days. Colonies were stained with a solution of $0.5 \%$ crystal violet in methanol.

\section{Quantification of DNA methylation by liquid chromatography-tandem mass spectrometry}

DNA was isolated using the Tissue \& Cell GenomicPrep MiniSpin Kit (GE Healthcare), quantified and subjected to hydrolysis with formic acid as previously described (12). UPLC/MS/MS analysis was carried out using a Waters XEVO TQ-S spectrometer at the 
Center of Scientific Instrumentation (University of Granada, Spain).

\section{Intracellular dNTP pool size determination}

dNTP levels were measured using a DNA polymerase assay with minor modifications (16-18). Extracts were incubated in $40 \mu \mathrm{L}$ of dUTPase buffer (34 mM Tris- $\mathrm{HCl}, \mathrm{pH} 7.8$, $5 \mathrm{mM} \mathrm{MgCl} 2$ ) or dUTPase buffer plus $30 \mathrm{ng}$ of human dUTPase (19) for 20 min at 37 ${ }^{\circ} \mathrm{C}$. To stop the reaction, samples were precipitated with methanol, centrifuged for 20 min at $16,000 \mathrm{~g}$, dried and used for the quantification of the dNTP pool size as described (12).

\section{Quantitative determination of uracil in DNA}

Uracil incorporation into DNA was measured using a qPCR-based assay following the protocol described by Horváth and Vértessy (2010) with minor variations $(20,21)$. Genomic DNA was extracted using MasterPure DNA Purification Kit (Epicentre), digested with SacI-HF (NE Biolabs) and 3-5 kb fragments containing the target template (GAPDH gene), isolated and purified from 1\% agarose gel. Two-fold dilution series of the DNA samples were amplified with PfuTurbo Hotstart (Agilent Technologies) or Taq polymerase (Bioline) and primers: 5'- CTC CTGCCCTTtGAGTTTGATG-3' and 5'- CAGCAGAGAAGCAGACAGTTATG-3'. qPCR was performed with CFX96 Real Time System C1000 Thermal Cycler (BioRad). $\mathrm{Cq}$ values obtained were used to determine uracil content as described (20). All values are referred to non-treated control siRNA-transfected cells.

\section{Immunofluorescence analysis of $\gamma-\mathrm{H} 2 \mathrm{AX}$ foci}

Cells were grown on sterile glass coverslips, fixed with $4 \%$ paraformaldehyde for 15 
min at RT, and permeabilized in PBS containing $0.1 \%$ Triton X-100 for 10 min at RT. Cells were then incubated with 1:250 diluted monoclonal anti- $\gamma \mathrm{H} 2 \mathrm{AX}$ antibody (clone JBW301, Millipore) for $30 \mathrm{~min}$ at $37^{\circ} \mathrm{C}$ and detected with secondary Alexa 488conjugated goat anti-mouse IgG (Invitrogen, Life Technologies). Coverslips were dehydrated in methanol and mounted in Vectashield with DAPI (Vector Laboratories, Inc.). Digital images were captured using a LEICA TCS SP5 confocal microscopy system and analyzed with FIJI/Image J.

\section{Statistics}

Results are expressed as mean \pm standard deviation of at least three independent replicates. One or two-way analysis of variance (ANOVA) followed by Dunnett's posthoc test (referred to control or non-treated cells) was used to analyze the data. Normality and homogeneity of variance assumptions were checked for each analysis. When the variances were different, Welch test followed by Games-Howell post-test were used. A Chi-square test was performed for $\gamma \mathrm{H} 2 \mathrm{AX}$-positive cells analysis. Fisher test was applied when the assumptions did not fulfill. Differences were considered significant when $P$-value was less than 0.05 . 


\section{RESULTS}

\section{DCTPP1 and dUTPase expression is up-regulated in response to decitabine}

We have previously shown that media supplementation with an excess of deoxycytidine induces an increase in the expression of DCTPP1 in HeLa cells (12). Hence, we expected that exposure to decitabine might induce a similar modulation of enzyme levels in response to the accumulation of aza-dCTP. As shown in Fig. 1, the amount of DCTPP1 protein increased up to three-fold at $24 \mathrm{~h}$ after addition to the medium of 10 $\mu \mathrm{M}$ aza-dCyd. At the same time, CDA, DCTD, TS and dUTPase expression was also up-regulated, suggesting the generation of metabolic intermediates and endpoints other than those leading to the formation of aza-dCTP.

\section{Depletion of DCTPP1 or dUTPase sensitizes cells to aza-dCyd.}

The observation that cell exposure to decitabine translates into the overexpression of two house-cleaning nucleotidohydrolases with capacity to hydrolyze non-canonical nucleotides, led us evaluate their potential protective role against the cytotoxic action of aza-dCyd once activated and incorporated into the nucleotide pool. HeLa cells were transfected with a siRNA pool against DCTPPI or DUT and then treated with increasing concentrations of the nucleoside analog for $24 \mathrm{~h}$. In all the experiments performed, a non-targeting siRNA pool was used as negative control. SiRNA-mediated depletion of the proteins was checked by Western blot (Fig. 2A). Strong downregulation of DCTPP1 or DUT led to increased toxicity by aza-dCyd (Fig. 2B). In contrast, cells transfected with the non-targeting siRNA pool did not exhibit a decrease in viability even in the presence of $1 \mathrm{mM}$ aza-dCyd. Similar results were obtained in siRNA knock-down experiments with the non-tumoral cell line MRC-5. The protective 
effect was decitabine-specific since the absence of DCTPP1 or dUTPase did not have a significant impact on cell proliferation after exposure to deoxycytidine or cytarabine.

DCTPP1 or dUTPase-deficient cells incubated with decitabine also displayed a reduced colony forming capacity compared to control cells (Fig. 2C). To investigate a potential resistance response to decitabine mediated by DCTPP1 and dUTPase, we tested whether the overexpression of these proteins might confer additional protection to aza-dCyd by comparing clonogenic survival of HeLa cells both parental and overproducing DCTPP1 or dUTPase after exposure to a range of decitabine doses (Fig. 3). At $1 \mu \mathrm{M}$ of aza-dCyd, the proportion of surviving cells expressing dUTPase was significantly enhanced compared to the number of viable colonies obtained with cells transfected with the empty vector. The overexpression of DCTPP1 had no impact on cell survival at any of the concentrations tested, suggesting that endogenous levels of DCTPP1 but not of dUTPase are sufficient to deal with potentially cytotoxic metabolites generated from aza-dCyd.

\section{DCTPP1 modulate DNA de-methylation induced by decitabine.}

Since DCTPP1 catalyzes the hydrolysis of $\mathrm{dCTP}$ to $\mathrm{dCMP}$ and pyrophosphate, we investigated its potential role in detoxification of aza-dCTP (activated form of decitabine). In vitro, DCTPP1 is active on aza-dCTP $\left(K_{\mathrm{m}}=54.04 \pm 0.34 \mu \mathrm{M}\right.$ and $k_{\text {cat }}=$ $\left.7.54 \pm 0.03 \mathrm{~s}^{-1}\right)$ (Fig. 4A), exhibiting kinetic parameters very similar to those obtained for $\operatorname{dCTP}\left(K_{\mathrm{m}}=47.63 \pm 2.66 \mu \mathrm{M}\right.$ and $\left.k_{\text {cat }}=5.69 \pm 0.18 \mathrm{~s}^{-1}\right)(12)$. The sigmoidal character of the aza-dCTP saturation curve and the double reciprocal plot showing nonlinear kinetics indicate that substrate binding is occurring with positive cooperativity $\left(\mathrm{n}_{\mathrm{H}}\right.$ $=2.29 \pm 0.03)($ Fig. $4 \mathrm{~A})$. No activity was detected with other nucleotide analogs tested such as ara-CTP (cytarabine triphosphate), dF-CTP (gemcitabine triphosphate) or ddCTP (zalcitabine triphosphate). 
The ability of DCTPP1 to act upon aza-dCTP suggests that under decitabine exposure, this enzyme may be preventing the accumulation of azacytosine in DNA and consequently the level of DNA methylation. To address this question, we determined changes in global methylation by measuring the ratio of 5-methylcytosine to cytosine by LC-MS/MS (Fig. 4B). Under our experimental conditions, we observed a significant decrease in global genome demethylation in control cells exposed to a dose of $100 \mu \mathrm{M}$ of decitabine for 48 hours. Depletion of DCTPP1 further increased the DNA demethylating activity of decitabine, which in the absence of the nucleotidohydrolase, caused significant reductions in the level of methylcytosine at 10 and $100 \mu \mathrm{M}$ concentrations. The methylation data support a role for DCTPP1 in preventing the incorporation of azacytosine in cell treatments with decitabine.

\section{Decitabine treatment alters the nucleotide pool composition}

It has been suggested that deaminated derivatives of aza-dCyd could interfere with the pyrimidine biosynthesis pathway by inhibiting TS (22). To investigate whether exposure to decitabine promotes the expansion of the dUTP pool in a similar way to other TS inhibitors such as 5-fluorouracil (23), we measured perturbations in the nucleotide pool upon treatment with aza-dCyd and the role of the nucleotidohydrolases DCTPP1 and dUTPase in these potential perturbations (Fig. 5).

The DNA polymerase-based assay used to quantify dNTPs does not allow discrimination between $\mathrm{dCTP}$ and aza-dCTP nor dUTP and aza-dUTP so we will refer to them as (aza-)dCTP or (aza-)dUTP. In control cells, only the treatment with the highest dose of decitabine induced an important increase in the concentration of intracellular (aza-)dCTP (from 3.9 to 4.1 and $8.2 \mathrm{pmol} / 10^{6}$ cells at $10 \mu \mathrm{M}$ or $100 \mu \mathrm{M}$ respectively) probably due to the conversion of aza-dCyd to aza-dCTP and the 
saturation of catabolic activities. On the other hand, the depletion of DCTPP1 promoted a substantial accumulation of aza-(dCTP) by treatment with decitabine (from 5.6 to 13.8 and $17.3 \mathrm{pmol} / 10^{6}$ cells) suggesting that DCTPP1 is likely the main catabolic activity of the activated form of decitabine. Unexpectedly, in the absence of decitabine, DCTPP1silenced cells also exhibited a significant pool of dUTP $\left(0.9 \mathrm{pmol} / 10^{6}\right.$ cells $)$ which cannot be detected in control cells. This pool increased by two-fold after exposure to $100 \mu \mathrm{M}$ of decitabine $\left(1.9 \mathrm{pmol} / 10^{6}\right.$ cells $)$. We hypothesize that the excess of dUTP might be generated as a result of the allosteric activation of dCMP deaminase by the abnormally high levels of $\mathrm{dCTP}$ that accumulate in these cells (12) rendering an excess of dUMP that is subsequently converted to dUTP.

In the case of cells subjected to siRNA-mediated depletion of dUTPase and exposed to aza-dCyd, important alterations in the nucleotide pool were observed. These alterations consisted in a strong increase in the intracellular amount of pyrimidine nucleotides: (aza-)dCTP (from 4.5 to 7.3 and $9.6 \mathrm{pmol} / 10^{6}$ cells), dTTP (from 8.6 to 12.1 and 11.5 $\mathrm{pmol} / 10^{6}$ cells) and (aza-)dUTP (from 0.4 to 1.6 and $2.2 \mathrm{pmol} / 10^{6}$ cells). While it is true that the expansion of the $\mathrm{dCTP}$ pool induced by decitabine can be mostly attributed to the formation of aza-dCTP, the increase of dTTP pools might be the consequence of an up-regulation of dNTP synthesis in response to massive DNA damage as reported for other genotoxic agents (24). Indeed, in DNA-damage-stressed cells, the synthesis of dTTP needed for recovery from DNA damage has been reported to be essentially mediated by TK (24). In spite of such expanded dTTP pool, decitabine exposure caused a two-fold increase in the $\mathrm{dUTP} /[\mathrm{dUTP}+\mathrm{dTTP}]$ ratio that elevates substantially the risk of uracil misincorporation into DNA.

Assessment of uracil and DNA damage induced by aza-dCyd in DCTPP1- and 


\section{dUTPase-deficient backgrounds}

An expected consequence of the accumulation of dUTP in the nucleotide pool as a result of the treatment with decitabine would be the misincorporation of uracil into DNA, especially in the absence of dUTPase. To monitor changes in the uracil genome content in the genome, we have used the real-time PCR assay described by Horvath and Vertessy (20) which utilizes the B-type DNA polymerase of Pyrococcus furiosus (Pfu) and the Taq DNA polymerase for amplifications. The Pfu/Taq PCR method is based on the fact that the DNA polymerase Pfu is strongly inhibited by uracil-containing DNA, whereas Taq DNA polymerase can replicate through the deaminated base. The differences in product formation by the two enzymes provides a mean to quantify the amount of uracil in the DNA sample (25). Genomic DNA was obtained from HeLa cells transfected with control, DCTPP1 or DUT siRNAs and treated with $0,1,10$ and 100 $\mu \mathrm{M}$ of aza-dCyd for 24 or 48 hours. Treatment of control cells with aza-dCyd for 24 hours produced a significant increase in the DNA uracil content which was further enhanced after exposure to the nucleoside analog for 48 hours $(P<0.00001)$ (Fig. 6A). We next analyzed the presence of uracil in DCTPP1- or dUTPase-silenced cells. Consistently with the existence of an endogenous dUTP pool, DCTPP1-depleted cells also exhibit higher constitutive levels of uracil in their genomic DNA. Concurrently with the expansion of the dUTP pool shown in Fig. 5, DCTPP1-deficient cells exposed to aza-dCyd also accumulated higher amounts of genomic uracil than control cells.

Similarly to DCTPP1, the lack of dUTPase provoked an increase in the basal levels of uracil in DNA, supporting the notion that dUTP is being constantly generated by the normal metabolism of the cell and removed by dUTPase from the nucleotide pool, which this way avoids its misincorporation into DNA. DUT-silenced cells were highly susceptible to accumulate uracil upon decitabine treatment, suggesting a major role for 
this enzyme in counteracting the formation of aza-dUTP.

Former studies have reported that cell treatment with aza-dCyd leads to the formation of DNA double-strand breaks (DSBs) in a direct or indirect manner and also promotes the accumulation of $\gamma \mathrm{H} 2 \mathrm{AX}$ foci (H2AX phosphoserine-139), a hallmark of the cellular response to DSBs (7). We proceeded to monitor $\gamma \mathrm{H} 2 \mathrm{AX}$ foci by immunofluorescence microscopy in DCTPP1 and dUTPase-depleted HeLa cells after incubation with decitabine (Fig. 6B). Control cells exposed to $10 \mu \mathrm{M}$ of aza-dCyd exhibited $7 \%$ of the population with $\gamma \mathrm{H} 2 \mathrm{AX}$ foci, while the percentage increased up to $10 \%$ and $12 \%$ in the absence of DCTPP1 and dUTPase respectively. Significantly, the number of $\gamma \mathrm{H} 2 \mathrm{AX}$ positive cells induced by $100 \mu \mathrm{M}$ aza-dCyd raised from $21 \%$ in control cells to $33 \%$ and 38\% in DCTPP1- and dUTPase-silenced cells respectively, observation that correlates with the notion that DNA breaks are a consequence of the incorporation of uracil or azauracil during replication. 


\section{DISCUSSION}

Catabolic activities may influence metabolism and thereby, the pharmacological efficacy of anticancer nucleoside analogs. Mammalian cells can uptake and incorporate decitabine into the nucleotide pool and be potentially channeled to different biochemical pathways (Fig. 7). Decitabine can be deaminated by CDA to aza-deoxyuridine, which is poorly phosphorylated by thymidine kinase (26) or alternatively, aza-dCyd can be converted into its active form via a phosphorylation pathway that includes $\mathrm{dCK}$, UMP/CMP kinase and NDPK. The ability of DCTPP1 to hydrolyze the activated form of decitabine and the hypersensitivity of DCTPP1-deficient cells exposed to this nucleoside analog suggest that this enzyme may be involved in a novel resistance pathway. Indeed, our data showing an increase in cytosine demethylation in DCTPP1silenced cells support the notion that this nucleotidohydrolase is interfering with the primary mode of action of decitabine and prevents the incorporation of aza-dCTP into DNA. Since the action of DCTPP1 entails the reversion of the phosphorylation steps involved in aza-dCyd activation, it is plausible that the relative activity ratio of the kinases UMP/CMP kinase and NDPK with regard to DCTPP1 may have predictive clinical value in treatments with this nucleoside analog.

Importantly, a number of evidences presented in this work suggest that aza-dCMP can also be deaminated by dCMP deaminase to produce aza-dUMP. Early reports have indeed demonstrated the formation of aza-dUMP in vivo after exposure to $\left[{ }^{3} \mathrm{H}\right] \mathrm{aza}-\mathrm{dCyd}$ (27). The presence of elevated levels of aza-dCTP generated as a result of decitabine metabolic activation would further favor deamination due to the allosteric activation of dCMP deaminase (28). In addition to the potential formation of aza-dUTP, it is likely that aza-dUMP itself acts as a competitive TS inhibitor and contributes this way to the 
accumulation of genotoxic dUTP in the nucleotide pool and the misincorporation of uracil in DNA. Numerous studies have established that the DNA damage associated to uracil-DNA is a significant mechanism of cytotoxicity induced by TS-inhibiting chemotherapeutic agents (23).

In agreement with this alternate genotoxic mechanism for decitabine, we have detected a significant amount of intracellular (aza-)dUTP after exposure to aza-dCyd, which correlated with an increased level of genomic uracil, especially in the absence of dUTPase or DCTPP1. While the dUTPase activity removes (aza-)dUTP from the nucleotide pool thus preserving genomic integrity, the absence of DCTPP1 might be promoting decitabine cytotoxicity by two different mechanisms: it removes aza-dCTP from the cellular pool and stimulates the synthesis of toxic aza-dUMP derivatives through the expansion of the intracellular concentration of (aza-)dCTP (12) and subsequent allosteric activation of dCMP deaminase (Fig. 7).

We postulate that decitabine enhances uracil misincorporation into DNA and activates base excision repair mechanisms. Indeed, a recent study has demonstrated that XRCC1 mutant cells, defective in BER, are hypersensitive to aza-dCyd and this phenotype is associated with an accumulation of abasic sites and DNA strand breaks in genomic DNA (11). It is therefore possible that some of these breaks are an indication of the formation of DNA repair intermediates induced by the presence of uracil and aza-uracil. In summary, the data from this work suggest a novel mode of action for decitabine in which aza-dCyd may ultimately lead to the toxic accumulation of (aza-)dUTP in the nucleotide pool and its incorporation into the genome. DCTPP1 and dUTPase would play an important role in this metabolic pathway and as potential factors affecting the clinical response to this demethylating agent, their expression levels may have predictive clinical value. Moreover, inhibition of these nucleotidohydrolases may 
constitute a novel approach to improve the efficacy of antitumor therapy with decitabine and circumvent potential drug resistance phenotypes. Indeed, novel molecules have been recently identified with capacity to specifically inhibit the activity of dUTPase and DCTPP1 that enhance the cytotoxic effect of pyrimidine anticancer agents $(29,30)$ thus highlighting the relevance of these nucleotidohydrolases in the mode of action of pyrimidine derivatives currently used in antitumour therapy.

\section{ACKNOWLEDGEMENTS}

We thank Aurora Constán for her technical assistance.

\section{AUTHOR'S CONTRIBUTIONS}

Conception and design: C.E. Requena, D. González-Pacanowska, A.E. Vidal, Development of methodology: C.E. Requena, A. Horváth, B.G. Vértessy Acquisition of data: C.E. Requena, G. Pérez-Moreno Analysis and interpretation of data: C.E. Requena, G. Pérez-Moreno, L.M. RuízPérez, D. González-Pacanowska, A.E. Vidal

Writing, review, and/or revision of the manuscript: C.E. Requena, A. Horváth, B.G. Vértessy, L.M. Ruíz-Pérez, D. González-Pacanowska, A.E. Vidal 


\section{REFERENCES}

1. Santos FP, Kantarjian H, Garcia-Manero G, Issa JP, Ravandi F. Decitabine in the treatment of myelodysplastic syndromes. Expert Rev Anticanc Ther 2010;10:922.

2. Griffiths EA, Gore SD. Epigenetic therapies in MDS and AML. Adv Exp Med Biol 2013;754:253-83.

3. Parker WB. Enzymology of purine and pyrimidine antimetabolites used in the treatment of cancer. Chem Rev 2009;109:2880-93.

4. Khan H, Vale C, Bhagat T, Verma A. Role of DNA methylation in the pathogenesis and treatment of myelodysplastic syndromes. Semin Hematol 2013;50:16-37.

5. Griffiths EA, Gore SD. DNA methyltransferase and histone deacetylase inhibitors in the treatment of myelodysplastic syndromes. Semin Hematol 2008;45:23-30.

6. Issa JP. Epigenetic changes in the myelodysplastic syndrome. Hematol Oncol Clin North Am 2010;24:317-30.

7. Palii SS, Van Emburgh BO, Sankpal UT, Brown KD, Robertson KD. DNA methylation inhibitor 5-Aza-2'-deoxycytidine induces reversible genome-wide DNA damage that is distinctly influenced by DNA methyltransferases 1 and 3B. Mol Cell Biol 2008;28:752-71.

8. Juttermann $\mathrm{R}$, $\mathrm{Li} \mathrm{E}$, Jaenisch $\mathrm{R}$. Toxicity of 5-aza-2'-deoxycytidine to mammalian cells is mediated primarily by covalent trapping of DNA methyltransferase rather than DNA demethylation. Proc Natl Acad Sci U S A 1994;91:11797-801.

9. Maslov AY, Lee M, Gundry M, Gravina S, Strogonova N, Tazearslan C, et al. 5aza-2'-deoxycytidine-induced genome rearrangements are mediated by DNMT1. Oncogene 2012;31:5172-9.

10. Jackson-Grusby L, Laird PW, Magge SN, Moeller BJ, Jaenisch R. Mutagenicity of 5-aza-2'-deoxycytidine is mediated by the mammalian DNA methyltransferase. Proc Natl Acad Sci U S A 1997;94:4681-5.

11. Orta ML, Hoglund A, Calderon-Montano JM, Dominguez I, Burgos-Moron E, Visnes T, et al. The PARP inhibitor Olaparib disrupts base excision repair of 5aza-2'-deoxycytidine lesions. Nucleic Acids Res 2014;42:9108-20.

12. Requena CE, Perez-Moreno G, Ruiz-Perez LM, Vidal AE, GonzalezPacanowska D. The NTP pyrophosphatase DCTPP1 contributes to the homoeostasis and cleansing of the dNTP pool in human cells. Biochem $\mathrm{J}$ 2014;459:171-80.

13. Ladner RD. The role of dUTPase and uracil-DNA repair in cancer chemotherapy. Curr Protein Pept Sci 2001;2:361-70.

14. Vertessy BG, Toth J. Keeping uracil out of DNA: physiological role, structure and catalytic mechanism of dUTPases. Acc Chem Res 2009;42:97-106.

15. Nagy GN, Leveles I, Vertessy BG. Preventive DNA repair by sanitizing the cellular (deoxy)nucleoside triphosphate pool. FEBS J 2014;281:4207-23.

16. Horowitz RW, Zhang H, Schwartz EL, Ladner RD, Wadler S. Measurement of deoxyuridine triphosphate and thymidine triphosphate in the extracts of 
thymidylate synthase-inhibited cells using a modified DNA polymerase assay. Biochem Pharmacol 1997;54:635-8.

17. Merenyi G, Kovari J, Toth J, Takacs E, Zagyva I, Erdei A, et al. Cellular response to efficient dUTPase RNAi silencing in stable HeLa cell lines perturbs expression levels of genes involved in thymidylate metabolism. Nucleos Nucleot Nucl 2011;30:369-90.

18. Hirmondo R, Szabo JE, Nyiri K, Tarjanyi S, Dobrotka P, Toth J, et al. Crossspecies inhibition of dUTPase via the Staphylococcal Stl protein perturbs dNTP pool and colony formation in Mycobacterium. DNA Repair (Amst) 2015;30:217.

19. Toth J, Varga B, Kovacs M, Malnasi-Csizmadia A, Vertessy BG. Kinetic mechanism of human dUTPase, an essential nucleotide pyrophosphatase enzyme. J Biol Chem 2007;282:33572-82.

20. Horvath A, Vertessy BG. A one-step method for quantitative determination of uracil in DNA by real-time PCR. Nucleic Acids Res 2010;38:e196.

21. Muha V, Horvath A, Bekesi A, Pukancsik M, Hodoscsek B, Merenyi G, et al. Uracil-containing DNA in Drosophila: stability, stage-specific accumulation, and developmental involvement. PLoS Genet 2012;8:e1002738.

22. Vesely J, Cihak A, Sorm F. Biochemical mechanisms of drug resistance. IX. Metabolic alterations in leukemic mouse cells following 5-aza-2'-deoxycytidine. Collect Czech Chem Commun 1969;34:901-9.

23. Wilson PM, Fazzone W, LaBonte MJ, Deng J, Neamati N, Ladner RD. Novel opportunities for thymidylate metabolism as a therapeutic target. Mol Cancer Ther 2008;7:3029-37.

24. Chen YL, Eriksson S, Chang ZF. Regulation and functional contribution of thymidine kinase 1 in repair of DNA damage. J Biol Chem 2010;285:27327-35.

25. Yan N, O'Day E, Wheeler LA, Engelman A, Lieberman J. HIV DNA is heavily uracilated, which protects it from autointegration. Proc Natl Acad Sci U S A;108:9244-9.

26. Munch-Petersen B, Cloos L, Tyrsted G, Eriksson S. Diverging substrate specificity of pure human thymidine kinases 1 and 2 against antiviral dideoxynucleosides. J Biol Chem 1991;266:9032-8.

27. Cihak A. Transformation of 5-aza-2'-deoxycytidine-3H and its incorporation in different systems of rapidly proliferating cells. Eur J Cancer 1978;14:117-24.

28. Momparler RL, Rossi M, Bouchard J, Bartolucci S, Momparler LF, Raia CA, et al. 5-AZA-2'-deoxycytidine synergistic action with thymidine on leukemic cells and interaction of 5-AZA-dCMP with dCMP deaminase. Adv Exp Med Biol 1986;195:157-63.

29. Saito K, Nagashima H, Noguchi K, Yoshisue K, Yokogawa T, Matsushima E, et al. First-in-human, phase I dose-escalation study of single and multiple doses of a first-in-class enhancer of fluoropyrimidines, a dUTPase inhibitor (TAS-114) in healthy male volunteers. Cancer Chemoth Pharm 2014;73:577-83.

30. Llona-Minguez S, Hoglund A, Jacques SA, Johansson L, Calderon-Montano JM, Claesson M, et al. Discovery of the First Potent and Selective Inhibitors of Human dCTP Pyrophosphatase 1. J Med Chem 2016 doi:10.1021/acs.jmedchem.5b01741. 


\section{FIGURE LEGENDS}

Fig. 1. Modulation of enzyme expression by aza-dCyd. HeLa cells were exposed to $10 \mu \mathrm{M}$ aza-dCyd and enzyme levels in $10 \mu \mathrm{g}$ of protein cell extract were determined by Western blot. Protein levels were quantified with ImageQuant image analysis software (GE Healthcare) and normalized to $\alpha$-tubulin. DCTPP1, dCTP pyrophosphatase 1; CDA, cytidine deaminase; DCK, deoxycytidine kinase; DCTD, dCMP deaminase; dUTPase, deoxyuridine triphosphate nucleotidohydrolase; TS, thymidylate synthase; TK, thymidine kinase; TMPK, thymidylate kinase. Expression at 24 hours was quantified and normalized to $\alpha$-tubulin before representation as a diagram bar.

Fig. 2. Down-regulation of DCTPP1 and dUTPase sensitizes cells to decitabine. A, Western blot analysis of HeLa and MRC-5 cells transfected with DCTPP1-specific siRNAs, DUT-specific siRNAs or a non-targeting (Control) siRNAs. Cell extracts were prepared at the fourth day post-transfection. Protein loading was normalized with antia-tubulin. B, HeLa and MRC-5 cells were exposed to the following nucleosides: decitabine (aza-dCyd); 2'-deoxycytidine (dCyd), and cytarabine (ara-Cyd). Percentage of viability was calculated relative to the number of viable cells in the absence of exogenous nucleosides. All values are mean $\pm \mathrm{SD}(\mathrm{N}=3)$. Data concerning the sensitivity of DCTPP1 siRNA-transfected cells to dCyd have been published elsewhere (12) and are included for comparison purposes. C, Clonogenic capacity of HeLa and MRC-5 cells transfected with DCTPP1-specific siRNA, DUT-specific siRNA or a nontargeting siRNA and treated with increasing doses of decitabine for $24 \mathrm{~h}$. All values are mean $\pm \mathrm{SD}(\mathrm{N}=4)$ 
Fig. 3. Effect of DCTPP1 and dUTPase overexpression on the clonogenic capacity of cells exposed to decitabine. Left panel, Western blot showing the levels of dUTPase and DCTPP1 proteins in HeLa cells transfected with pcDNA3-DCTPP1 (DCTPP1 OE), pcDNA3-DUT (DUT OE) or the empty pcDNA3 vector (Control). Right panel, Clonogenic survival of HeLa cells expressing normal (Control) or increased levels of DCTPP1 (DCTPP1 OE) or dUTPase (DUT OE) and treated with increasing doses of decitabine for $24 \mathrm{~h}$. All values are mean $\pm \mathrm{SD}(\mathrm{N}=3)$.

Fig. 4. DCTPP1 catalyzes the hydrolysis of aza-dCTP and prevents DNA demethylation in human cells. A, Left panel, The enzymatic assay was performed by incubating $0.5 \mu \mathrm{M}$ of DCTPP1 with increasing concentrations of aza-dCTP (20-250 $\mu \mathrm{M})$ under optimized reaction conditions as previously described (12). Kinetic parameters and the Hill's coefficient $\left(\mathrm{n}_{\mathrm{H}}\right)$ resulted from adjusting the data to the Hill's equation. All values are mean $\pm \mathrm{SD}(\mathrm{N}=3)$. Right panel, Double reciprocal plot showing non-linear kinetics for aza-dCTP. B, Global DNA methylation after supplementation of HeLa cells with $0-100 \mu \mathrm{M}$ of aza-dCyd for 24 or $48 \mathrm{~h}$. All values are mean $\pm \mathrm{SD}(\mathrm{N}=3)$.

Fig. 5. Effect of decitabine on the intracellular dNTP pool. Nucleotide concentration was measured in HeLa cells transfected with non-targeting, DCTPP1 or DUT-specific siRNA pools. Treatments were carried out with $0-100 \mu \mathrm{M}$ of decitabine for $24 \mathrm{~h}$. All values are mean $\pm \mathrm{SD}(\mathrm{N}=4)$.

Fig. 6. Decitabine induces uracil accumulation and DNA strand breaks specially in the absence of DCTPP1 or dUTPase. A, Uracil content in genomic DNA from HeLa cells transfected with Control, DCTPP1 or DUT siRNAs and treated with $0-100 \mu \mathrm{M}$ 
aza-dCyd for 24 or $48 \mathrm{~h}$. Values resulting from genomic DNA isolated from non-treated Control siRNA-transfected cells were used as reference. All values are mean \pm SD $(\mathrm{N}=3)$. $P$-values underlined with dotted line are referred to treated Control siRNA cells. $\mathrm{B}$, Left panel, Representative microscopy images showing $\gamma \mathrm{H} 2 \mathrm{AX}$ staining in nontreated or $10 \mu \mathrm{M}$ and $100 \mu \mathrm{M}$ aza-dCyd-treated HeLa cells. All chosen fields contain a similar number of cells as determined by DAPI staining (not shown). Right panel, Percentage plots of $\gamma \mathrm{H} 2 \mathrm{AX}$-positive cells in Control, DCTPP1, and DUT siRNA cells treated with aza-dCyd. $\gamma \mathrm{H} 2 \mathrm{AX}$-positive cells ( $>5$ foci) were scored from a total of two hundred cells per cell line and dose.

Fig. 7. Role of DCTPP1 and dUTPase in the response to decitabine. Aza-dCyd is converted into its active form via a phosphorylation pathway initiated by deoxycytidine kinase (dCK). DCTPP1 can hydrolyze aza-dCTP and could prevent its incorporation into DNA and the formation of DNMT-DNA cross-links. Alternatively, aza-dCyd can be deactivated by cytidine deaminase (CDA) to aza-deoxyuridine (aza-dUrd) which is poorly phosphorylated by thymidine kinase (TK). The aza-dCMP intermediate can also be deaminated by dCMP deaminase (DCTD) to produce aza-dUMP, which might potentially inhibit thymidylate synthase (TS). The accumulation of genotoxic (aza-)dUTP would promote uracil misincorporation and DNA damage. 